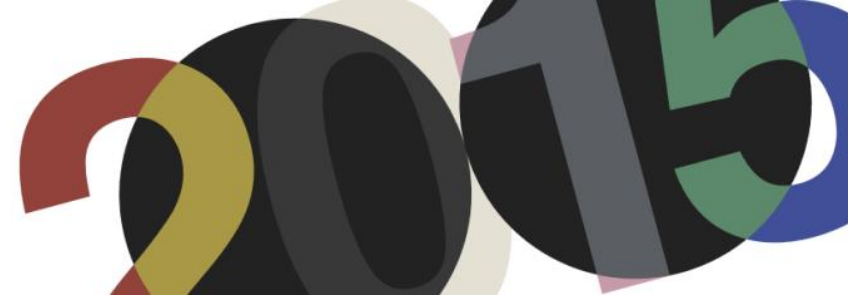

DOI: http://dx.doi.org/10.4995/LC2015.2015.642

\title{
Team10 out of CIAM: Sobre el papel de Le Corbusier. Identificación y legado
}

\author{
A. Campos Uribe
}

Escuela Técnica Superior de Arquitectura de Valencia

\begin{abstract}
Resumen: Aunque existen diversas versiones sobre el nacimiento del Team10 y la disolución de los CIAM, todas coinciden en el carácter de ruptura. Frente a la versión canónica que explica el fin de los CIAM como confrontación, este artículo muestra, a través del estudio de los documentos que pasaron por el estudio de Le Corbusier, que muchas de las ideas del grupo ya existían en el interior de los CIAM, y que la creación del Team10 contó con el apoyo de los miembros fundadores. Es especialmente importante el apoyo personal de Le Corbusier que a través de cartas y dibujos entendió muy pronto que era el momento de apartarse y dejar paso a la nueva generación; hasta el punto de constatar que sin su apoyo es posible que el Team10 nunca hubiera sido creado. Le Corbusier, como figura y ejemplo de los jóvenes que supo, generosamente, dar el relevo a tiempo.
\end{abstract}

Abstract: Albeit different versions of Team 10 birth and dissolution of CIAM exist, all of them coincide in its disruptive nature. This article tries to prove, far from the canonical representation of the end of CIAM and by studying the documents that went through Le Corbusier's atelier in Paris; that many of the ideas of Team 10 were already present inside CIAM and that Team 10's creation had the support of several of CIAM's founding members. It is especially important Le Corbusier's personal backing. Le Corbusier, who through letters and drawings understood very soon that it was the time to step aside and give way to the new generation. In fact, it was vital to the creation of Team 10, without whom it would not have been possible.

Palabras Clave: CIAM; Team 10; Le Corbusier; Aix-en-Provence; Dubrovnik; Smithson, Bakema.

Keywords: CIAM; Team 10; Le Corbusier; Aix-en-Provence; Dubrovnik; Smithson, Bakema.

\section{Introducción}

Aunque existen diversas versiones sobre el nacimiento del Team10, la mayoría coinciden en su carácter de ruptura. En los CIAM de post-guerra la metodología reflejada en la Charte d'Athènes, comenzaba a levantar críticas de las generaciones más jóvenes. Un inesperado dogmatismo se imponían entre los miembros de la segunda generación, alineados con la interpretación exhaustiva de la carta en cuanto a la separación de funciones y el enfoque analítico; mientras los miembros fundadores, verdaderos propulsores del espíritu de los CIAM, iban abandonando progresivamente su participación activa en los congresos. La tercera generación se nutriría de multitud de ideas que habían comenzado a surgir bajo el paraguas del CIAMVI en Bridgwater (1947) y que representaban un nuevo enfoque en el que la importancia se trasladaba a la imaginación y la síntesis, al concepto de identidad, y en particular a las relaciones entre funciones más que las funciones en sí mismas.

Como explica Annie Pedret en su trabajo titulado "CIAM and the emergence of Team10 thinking", en realidad el pensamiento del Team10 tiene un marcado carácter de continuidad con las ideas que habían nacido en los últimos congresos. Pedret conecta las propuestas teóricas del Team10 con conceptos promovidos desde 1947 por los grupos holandeses (Opbouw y de 8) e inglés (Mars), pero también por personas como Alfred Roth o Sigfrid

\footnotetext{
${ }^{1}$ Pedret, Annie. CIAM and the emergence of Team 10 thinking 1945-1959, Tesis Inédita defendida el 06/2001 en el Massachusetts Institute of Tecnhology (A partir de ahora AP)
} 
Giedion, o el grupo italiano del que destaca Ernesto N. Rogers; demostrando que los conceptos de identity, doorstep, the art of living, el visual group o el cluster, el change and growth y en general la apuesta por una arquitectura de relaciones, no son patrimonio del Team10. El trabajo nos permite entender el grupo como la continuación, que no ruptura, de los CIAM aunque con un nombre distinto, y comprender que ese cambio de nombre fue en realidad un acontecimiento casual forzado por personas como Peter Smithson o John Voelcker, que nunca creyeron que en la misma organización fuera posible dar un verdadero giro de ideas.

Resulta muy interesante preguntarse por el papel de Le Corbusier durante los últimos congresos, y en particular por su relación con los actores que posteriormente formaron el Team10. En un primer momento Le Corbusier trató de monopolizar el pensamiento de los CIAM2, pero tras la guerra y ante las reinvindicaciones en el seno de la organización entendió que era hora de dejar paso a la generación de los jóvenes para que "apoyados sobre las espaldas" de los miembros fundadores emprendieran su propio camino. A partir de ese momento Le Corbusier resultó ser uno de los más importantes aliados de la nueva generación y en numerosas ocasiones intervino para otorgarles su confianza; hasta el punto de poder afirmar que el nacimiento del Team10 y su "ruptura" con los CIAM no hubiera sido posible sin su apoyo explícito y enormemente generoso.

Aunque hay una versión distinta del nacimiento del Team10 contada por cada uno de sus miembros, mediante el análisis de los documentos que circularon por el estudio de Le Corbusier podremos ponerlas a prueba para encontrar el verdadero papel que tuvo en la creación del grupo.

\section{El futuro de CIAM: los jóvenes toman el relevo}

A lo largo de los años de guerra, el pensamiento de los CIAM se había desarrollado en direcciones contrarias. Por una parte Le Corbusier y Sert promovían el urbanismo de la "Charte d'Athènes", mientras Giedion y Alfred Roth asentaban las bases de un "post-funcionalismo" regional, social y cultural. En su libro New Architecture, que influenció mucho a la nueva generación ${ }^{4}$, Roth aportaba ideas nuevas: la historia como herramienta para comprender el presente e inventar el futuro, el valor de las relaciones humanas en la arquitectura o la importancia de la imaginación. Giedion, en Space, Time, and Architecture ${ }^{5}$, incidía también en la importancia de las "relaciones entre cosas" y de la historia, reforzando la necesidad de acercamiento a las culturas regionales. En esa misma línea se movía el pensamiento de los grupos holandeses Opbouw y De 8 de los que Jaap Bakema y Aldo van Eyck eran miembros, y del inglés Mars Group al que pertenecían los Smithson, Voelcker y Howell.

En el primer congreso tras la guerra, el CIAM VI en Bridgewater, ambos grupos realizan una importante contribución que sin embargo no sería recogida en los informes del congreso. En particular Aldo van Eyck, que había vivido en Suiza durante la guerra y compartía muchas ideas con Giedion, hace un alegato en contra de la tiranía del sentido común ${ }^{6}$, y a favor de un cambio de dirección en el enfoque excesivamente analítico y

\footnotetext{
${ }^{2}$ En muchas ocasiones Le Corbusier se encargó de elaborar el discurso oficial haciendo caso omiso de las verdaderas discusiones que se producían en los CIAM. Cambió en varias ocasiones el Consejo, nombrando a amigos como Sert o Giedion, asegurándose así el control de lo que sucedía

${ }^{3}$ Roth, Alfred. The New Architecture. Zurich: Les Editions A'Akrchitecture, 1940

${ }^{4}$ Según declaraciones de Peter y Alison Smithson, Giancarlo de Carlo, Aldo van Eyck y Ernesto Rogers

${ }^{5}$ Roth, Alfred. The New Architecture. Zurich: Les Editions A'Akrchitecture, 1940

${ }^{6}$ Entre sus palabras podemos encontrar por ejemplo la idea de que "a new consciuosness has begun to permeate mankind" $o$

"imagination is the essence of existence". También menciones a Giedion o a los logros de Le Corbusier, Mondrian o

Brancusi como ejemplos para la nueva dirección en los CIAM.
} 
deductivo de los CIAM que había llevado a una separación entre la arquitectura y las personas a las que finalmente servía. Las discusiones alrededor del término habitat son constantes dentro de CIAM, y los nuevos miembros apoyan una definición mucho más extensa e inclusiva, mientras que los veteranos son reacios a cambiar la teoría construida a lo largo de los CIAM previos a la guerra. En sucesivos congresos y encuentros, la joven generación no cesa de proponer nuevas ideas para una arquitectura y un urbanismo más humanos, y sin embargo los informes de resultados de las reuniones seguirían ignorando sus reivindicaciones hasta 1952.

En la reunión del consejo de los CIAM de mayo del $52^{7}$, en la que están presentes Candilis, Gropius, Rogers y Giedion, se produce la primera mención directa de Le Corbusier a los jóvenes. La reunión se realiza con el objetivo de decidir el futuro de los CIAM y el tipo de participación de los jeunes en la organización. Le Corbusier afirma que los fundadores ya son demasiado viejos y no tienen la flexibilidad necesaria para responder a los nuevos requerimientos. Sin embargo, está de acuerdo con Gropius en que una salida de los anciens ahora sería muy teatral, y a la vista de que los jóvenes únicamente piden una mayor participación (“les jeunes ne se sentent pas prêts à agir seuls"), se decide darles un papel más activo en el próximo congreso en Aix-enProvence. Ya en 1952 había planes para dar el relevo a los jeunes, y fueron Gropius y Le Corbusier los que propusieron al CIAM Council que se hicieran cargo de la organización del décimo congreso.

Es especialmente esclarecedora la carta que envía Sert a Le Corbusier ${ }^{8}$ en la que explica su punto vista sobre el futuro de los CIAM. Propone que no haya diferenciación alguna entre jóvenes o viejos, pues la tarea de los CIAM no es de una generación sino que debería continuarse en la siguiente. "Nous avons aussi passé beaucoup de temps à des controverses philosophiques. Aujourd'hui nous devons agir, faire face aux réalités”. Hace además una serie de sugerencias para la reorganización de los Congresos: limitar el número de miembros, restaurar la estructura anterior a la guerra (CIRPAC) y celebrar un congreso cada dos años dando la responsabilidad de su organización a los jóvenes. Estas propuestas, nacidas de una empatía especial de Sert por los jeunes, serán en su mayoría llevadas a cabo en el período de tiempo desde esa primera reunión en el 52 hasta la disolución definitiva de los CIAM en 1959.

En Aix-en-Provence los grupos participan más que nunca ${ }^{9}$ y se crea un comité de transferencia (Figl). Aix-enProvence será el primer congreso al que asisten los Smithson, y en el que presentan su conocido "Urban ReIdentification Grid", introduciendo la idea de las escalas de asociación o la de "doorstep", y será la primera ocasión en la que un grupo de jóvenes encuentra una misma manera de trabajar que los unirá hasta 1981 bajo el nombre de Team10. En Aix se reconoce un salto generacional para algunos irreconciliable y de que los CIAM, como organización, es incapaz de evolucionar.

(pg 32-43) Van Eyck, Aldo. Collected Articles and Other Writings 1947-1998. Amsterdam: Vincent Ligtelijn y Francis Strauven, SUN Publishers, 2008

${ }^{7}$ Conseil CIAM Mai 1952. (Fondation Le Corbusier - FLC D3-1 2-8)

${ }^{8}$ J.L. Sert 26/10/1952 a Le Corbusier. (FLC D3-1 538-543)

${ }^{9}$ J.L-Sert 14/07/1952 (FLC D3-1 553) 


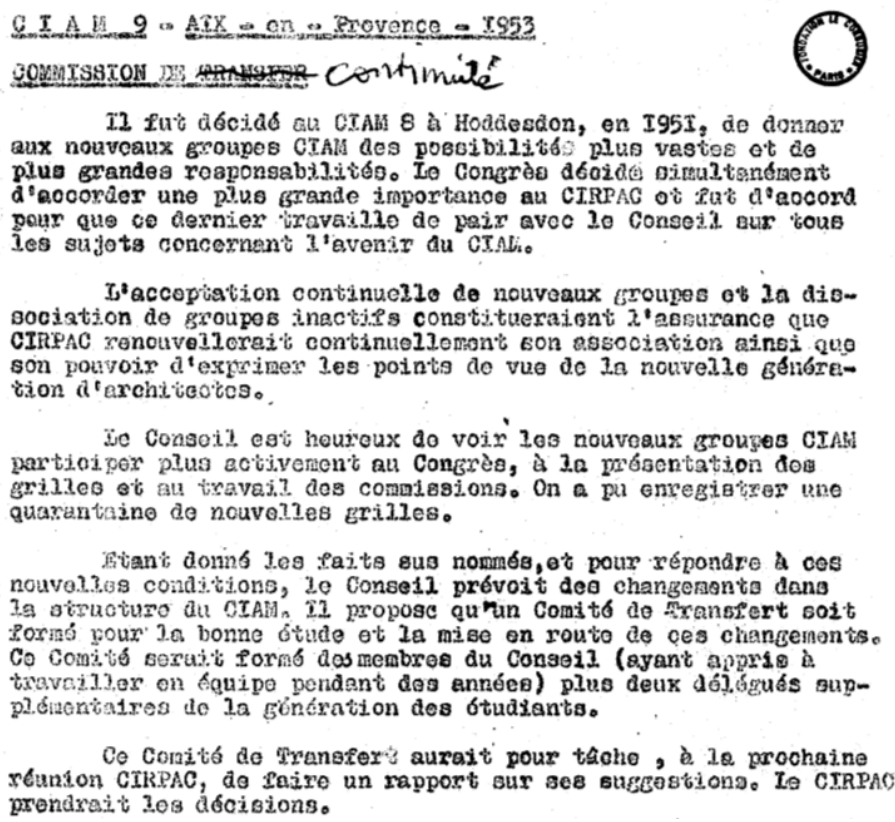

1. En la copia recibida por Le Corbusier, aparece cambiado Comission de Transfer por Comission de Continuité. Esto explica su postura al respecto. 24/07/1953 (FLC D3-1 553) OFLC-ADAGP

\section{El manifiesto de Doorn}

Tras el congreso, en la reunión de Doorn, los jóvenes alcanzan un acuerdo en torno al concepto de habitat. En la redacción del documento final, que se llamó "Statement on Habitat" aparece por primera vez el famoso diagrama de Geddes (Fig 2) y el concepto de "Scale of Association". Se constata el valor de la Carta de Atenas pero se reconocen sus limitaciones, proponiendo un método que libere el potencial acotado por las cuatro funciones; y se pasa a considerar cada comunidad como un caso concreto que hay que tratar con sus peculiaridades (particular total complex $)^{10}$. En esa misma reunión se habla de la organización de los CIAM. Bakema y Van Eyck están a favor de la continuidad (Fig3), y sugerieren al consejo que los jóvenes organicen CIAMX ${ }^{11}$.

En la reunión del Consejo y los delegados en $1954^{12}$, con Le Corbusier y la mayoría de los miembros del Team10 presentes, se observa una primera incursión de las nuevas ideas en la organización. Se puede extraer de la transcripción que los grupos Mars (Lasdun+Smithson+Voelcker), Opbouw (Bakema), Gamma (Candilis) y De 8 (Van Eyck) toman una parte muy activa en el encuentro. Se acuerda en la reunión que la preparación del CIAMX se encargará a un grupo pequeño de individuos (Bakema, Smithson, Candilis y Gutman), que el tema del congreso sería el habitat humano y por tanto el trabajo presentado reflejaría el habitat ideal teniendo en cuenta las condiciones climáticas y sociales (Mars Group), y que la síntesis era más importante que ampliar más el análisis (Van Eyck y Giedion).

\footnotetext{
${ }^{10}$ This method of work will induce a study of human association as a first principle, and of the four functions as aspects of each total problem. (FLC D2-8-13) El Statement on Habitat sería renombrado por Alison Smithson como The Doorn Manifesto, como base para el desarrollo teórico del Team10. Como constata Pedret, la redacción del documento fue manipulada por los Smithson para construir la idea de que ellos eran los autores (AP 56)

${ }^{11}$ Discussion on organizational maters Doorn 29/01/1954

(ET10 25-27) Smithson, Alison. The Emergence of Team 10 out of CIAM. London: Architectural Association, 1982

12 (FLC D2-8 142-144)
} 

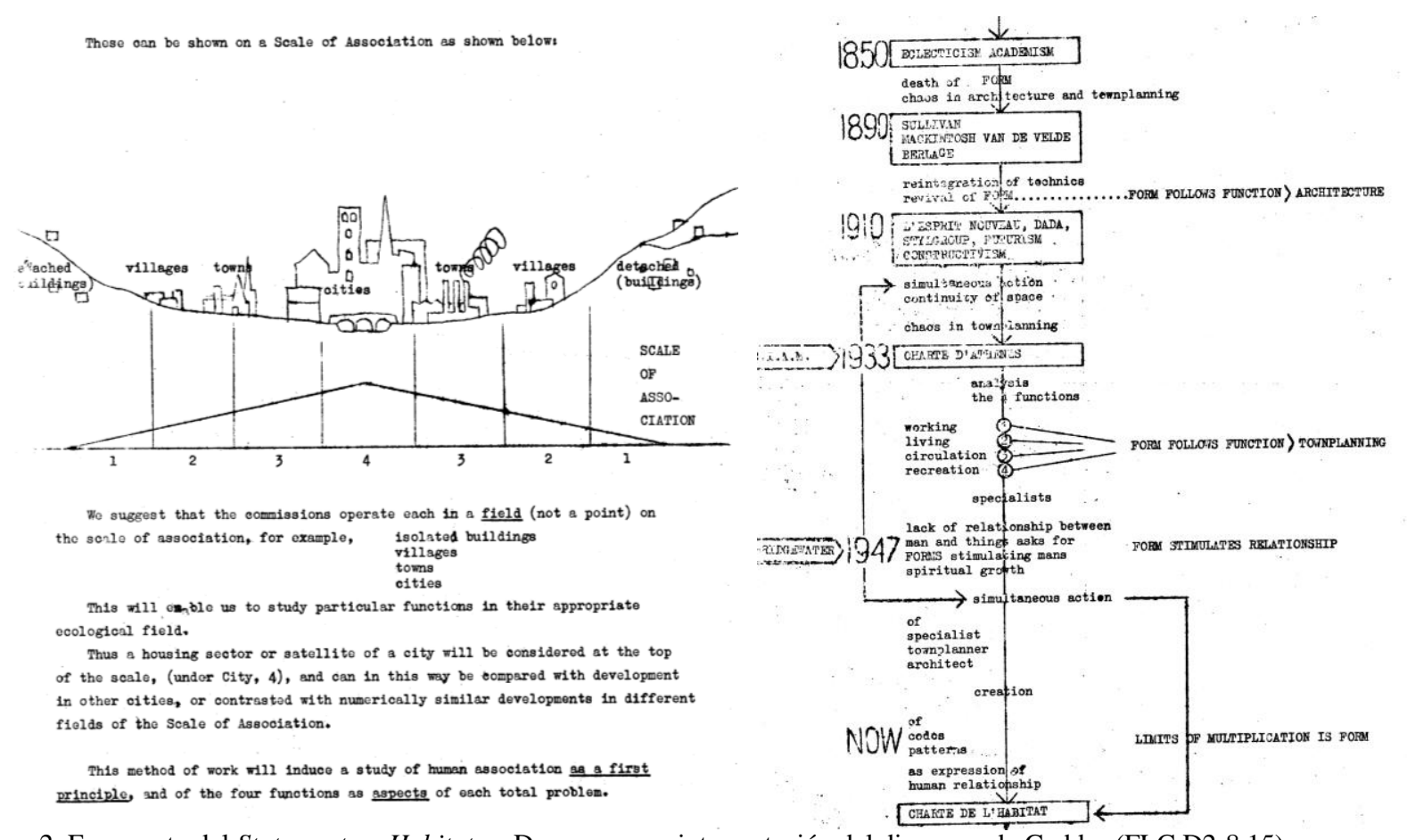

2. Fragmento del Statement on Habitat en Doorn, con su interpretación del diagrama de Geddes (FLC D2-8 15)

3. Esquema elaborado por Bakema (FLC D2-8 16) CFLC-ADAGP

\section{Crisis en el Team 10: "if you want to continue, continue in your own way"}

A lo largo de los primeros meses de preparación del congreso y discusión sobre el término habitat, se encuentran las primeras diferencias entre los jóvenes que a punto están de terminar con el grupo antes de haberse creado. Tras la reunión de Doorn, Peter Smithson es el encargado de redactar el marco del congreso y las instrucciones para los grupos. En el proceso de redacción, mediante sucesivas reuniones, se abandonan algunos de los acuerdos de Doorn y se modifican o añaden otros ${ }^{13}$. El resultado, según Sert, Gropius y Giedion, es un tanto ambiguo e impreciso, y critica duramente los logros previos de la organización ${ }^{14}$. Bakema hace una serie sugerencias para realizar modificaciones que son ignoradas ${ }^{15}$, y finalmente toma la decisión de redactar un documento que se conoce por Orientation ${ }^{16}$ y enviarlo a todos los miembros de los CIAM. Smithson, Howell y

\footnotetext{
${ }^{13}$ El documento final presentado al consejo se conoce como Draft Framework 3. Instructions to groups. (FLC D2-8 36-39)

${ }^{14}$ Tres documentos muestran la preocupación por la falta de precisión. Una carta a Bakema (FLC D3-7 130) de Gropius, Giedion y Sert en la que exigen claridad ("we received a program that has many fine statements of a general type, but is lacking in precise directives") y muestran su enfado por la crítica ("it is not customary in our congress to start criticising past efforts"). Una carta de Giedion a Le Corbusier (FLC D3-7 128) "l'ataque injuste vis-à-vis de la Charte d'Athenes", "le manque de precision". El último, de Candilis, es una carta a Smithson y Bakema en la que afirma que Giedión está "terriblement inquiet au sujet de l'organisation spirituelle du Congrès" (FLC D3-7 135). Smithson responderá enfadado: "What do the Professors want?" (ET10 43)

${ }^{15}$ Carta de Bakema 22/09/1954, en la que atiende a las exigencias de los veteranos. Propone también incluir el concepto de "the art of living", el trabajo con especialistas diversos, el concepto de "identity" y el de "change and growth", la importancia de la búsqueda de nuevos patrones... Todos vitales para comprender el pensamiento del Team10, ya reivindicados por los holandeses. (FLC D2-8 43-45)

${ }^{16}$ Redactado por Bakema 24/10/1954 con la ayuda de Van Eyck en el que proponen un marco específico para el congreso. Se organiza en dos grandes partes. En "Orientation" se hace una narración histórica del momento y se reconocen los logros de los CIAM, apoyando la continuidad. En "Organisation" se recoge el documento acordado en Doorn y se desarrolla de forma
} 
Voelcker responderán enfadados a Bakema $^{17}$ e intentarán aclarar la situación, pero la diferencia de criterios llevará finalmente a la intervención de Le Corbusier y Sert.

En Orientation el Dutch Group ponía más énfasis en las relaciones ente personas y cosas, y marcaba cuatro ejes para organizar el congreso. "The Aesthetics of number", que pretende solucionar la monotonía producida por la estandarización y la repetición en grandes actuaciones. "Growth and change" (Bakema) el habitat no debe resistirse al desarrollo y cambio de aquellos a los que sirve. "The greater reality of the doorstep" (Van Eyck) que pretende desarrollar las relaciones "between man and man, between man and things", y superar las polaridades existentes (individual-colectivo, físico-espiritual, dentro-fuera, parte-todo), entendiendo que son "dualphenomenon" ( anteriores, que trabaja sobre la Scale of Association mediante el estudio de proyectos concretos en su contexto. Se pretende establecer un marco teórico y posteriormente estudiar los proyectos bajo el diagrama de Geddes, por lo que no se abandona lo propuesto por Mars. El Mars Group, con su enfoque empírico, pretendía que ese marco teórico se desarrollara a través del estudio de proyectos, pues creían que proponerlo desde el principio supondría acotar las ideas. Todas las propuestas del Team10 en torno al término habitat tratan de dar respuesta a unas necesidades que ya no son para un ser humano abstracto sino concretas para cada comunidad y cada individuo.

El siguiente contacto se produce en el apartamento en París de Le Corbusier en septiembre de 1954. Como recuerda Alison Smithson ${ }^{18}$, la reunión tenía un doble motivo: por una parte conocer personalmente a Le Corbusier, que estaba dispuesto a pasar la dirección de los CIAM a los jóvenes sin problemas, y por otra discutir el marco del décimo congreso (base de todas las propuestas posteriores del Team10) que tenía como objetivo regenerar los CIAM. Le Corbusier apoya las inquietudes del comité ("if you want to continue, continue in your own way") ${ }^{19}$ e incluso afirma ser un miembro más del grupo pero sin el deseo de que su figura pese sobre ellos ${ }^{20}$.

En los documentos de preparación para la reunión elaborados por Candilis se exponen las razones por las piensan que es necesario un cambio en los CIAM $^{21}$ y se menciona por primera vez el nombre "EquipeX" $X^{22}$, que

muy distinta al de los Smithson, más particularizada y concreta. Es un documento que permite entender el valor de las aportaciones del Dutch Group al pensamiento del Team10.

(FLC D2-8 47-55)

${ }^{17}$ En una carta a Bakema y firmada por Howell, A. and P. Smithson y Voelcker: "we are completely bewildered by the document of October 24th", "the author of the new sections has very largely missed the point of the last years work". En realidad, mediante el análisis de los documentos, no se encuentra un cambio sino más bien un desarrollo y concreción. (ET10 36-37)

${ }^{18}$ Smithson, Alison. Team Ten Meetings, 1953-1981. United States: Rizzoli International Publications, 1991

${ }^{19}$ Ibid

"According to Sandy van Ginkel, Le Corbusier said: 'If you want to continue, continue in your own way.,",

${ }^{20}$ Risselada, M. and Van den Heuvel, D. Team 10: in search of a Utopia of the present. Netherlands: NAI Publishers, 2006.

"Van Eyck: Team 10 started within CIAM, and there were people like Ernesto Rogers and Le Corbusier himself who were enthusiastic and supported us: some of our meetings took place at Le Corbusier's house, do you remember? So he was 'sur les épaules des autres', 'abbas les emmerdeurs' he wrote. Le Corbusier said: 'I am a member but I do not want to weigh on you', 'I belong to Team 10','

21 “Nous pensons, surtout après Aix, que le moment est venu de donner de l'eau de jouvence au mouvement CIAM". "La senilité, la fatigue, la mediocrité envahissent CIAM". "Les congrès deviennent avec le temps un lieu de rencontre"

En el documento se constata que el acuerdo alrededor del Draft3 no era tan extenso como Smithson afirmaba, pues Candilis expone muchas ideas que serán utilizadas en el documento "Orientation" como el carácter evolutivo del habitat y el concepto de "l'Habitat du grand nombre" demostrando que "Orientation" no era simplemente la opinión de los holandeses. (FLC D37 7-9)

${ }^{22}$ Team10 es el nombre que se da al grupo encargado de organizar CIAM X 


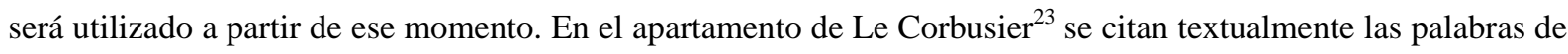
Candilis pero se minimiza la crítica a los CIAM. Le Corbusier da su opinión sobre la idea de habitat en la que distingue entre "the dwelling as a container: le dedans, and the extension of the dwelling: le dehors", en contraposición a la idea mucho más inclusiva de los jóvenes. También afirma que "dans les reserches [...] la primauté est donnée à l'humain est non pas à la mechanisation" y sugiere que no se utilicen nombre comunes para las agrupaciones de edificios, haciendo alusión a las escalas del Scale of Association (isolate, village, town, city); en su lugar propone utilizar simplemente "the Habitat in isolation, in Small Communities...". En el documento Orientation Bakema responde a esa petición, frente a los documentos de Smithson-Howell-Voelcker que se niegan a abandonar la Scale of Association. La idea de Le Corbusier es pasar el relevo a los jóvenes y que los mayores recapitulen los 25 años de los CIAM, así como la elaboración de una “Chartre de l'Habitat” con los resultados del décimo congreso.

Si bien es cierto que había personajes como Le Corbusier que veían una clara diferenciación en función de la generación, otros como Ernesto N. Rogers pensaban que la idea de separar a jeunes y anciens solo llevaba a la división y no a la unión según la vitalidad del espíritu. Miembros del Team10 también mostrarían su aprecio por figuras como la de Le Corbusier ${ }^{24}$, entendiendo que él no era el problema; sin dar importancia al salto generacional sino más bien criticando la deriva producida por individuos sin vitalidad ni objetivos. No creían en una separación entre un old y un new $C I A M^{25}$, sino que lo veían como una organización que ya no era útil para solucionar los nuevos interrogantes.

En cartas a Bakema Le Corbusier muestra su simpatía (“faites mes amitiés à tous, spécialement à Van Eyck pour qui j'ai, comme pour vous, beaucoup d'estime"). Sin embargo, a lo largo de 1955 se suceden las muestras de decepción de personajes como Emery o Wogenscky ${ }^{26}$. Desde Cap Martin ${ }^{27}$ Le Corbusier muestra su molestia con los jóvenes y cree que hay una atmósfera equivocada (“les joueurs jouant”). Piensa que “les jeunes n'ont pas la force d'embrasser la térrible complexité du phénomène moderne", y cree que su tarea es "prouver par des oeuvres!" y la de los veteranos redactar la Charte de l'Habitat que ha de "servir aux jeunes, de règle, de consigne, d'arme d'attaque et de défense".

\footnotetext{
${ }^{23}$ Meeting of Team X with the Vice-Presiden and Secretary General of CIAM.

Team X (Bakema, Van Eyck, Van Ginkel, Smithsons, Howells y Candilis), Le Corbusier y Giedion. (FLC D2-8 40-42)

${ }^{24}$ Encontramos menciones a Le Corbusier en muchas declaraciones del Team10.

Por ejemplo Van Eyck en su intervención en CIAMVI ("the achievement of men like Le Corbusier, Mondrian or Brancusi compels us to believe that we are indeed approaching a brighter era")

${ }^{25}$ Jaap Bakema y Giancarlo de Carlo hablan sobre Le Corbusier en Otterlo 1959

(Newman, Oscar, CIAM '59 in Otterlo. New York: Universe Books Inc, 1961)

Bakema: "I don't agree with those who speak of an old CIAM and a new CIAM. There can never be an old and a new: we simply go on"

La intervención de Giancarlo es especialmente interesante por analizar los CIAM subrayando la excepcionalidad de la figura de Le Corbusier. ("Le Corbusier, endowed with a boundless formal shrewdness and with an unrelenting technical earnestness, always managed to avoid the bottomless pit of academicism")

${ }^{26}$ En una carta del 6 de abril de 1955 de Wogenscky a Emery, copia enviada a Le Corbusier, Wog da su opinión y muestra su decepción (“je suis eidéré de voir la pauvreté, la partialité, l'aspect négatif et destucteur, l'absence de propositions concretes... qui caractérisent à mon avis le programme préparé par le groupe designé. C'est une déception car j'attendais infiniment mieux de Bakema et de Van Eyck"). La carta aparece anotada a mano con la respuesta de Le Corbusier. (FLC D2-8 331-332)

${ }^{27}$ Ibid
} 


\section{La intervención de Le Corbusier}

Empujado por una carta personal de Giedion muy crítica con el Team $10^{28}$, en la que pedía su intervención directa, Le Corbusier se reunirá el 9 de mayo de $1955^{29}$ con Candilis, que aclara el punto de vista de los jóvenes. Le Corbusier envía el documento "Intervention du Vice-President pour l'Europe" ${ }^{\prime 30}$ a todos los miembros de los CIAM, defendiendo el enfoque y el trabajo realizado por el Team10. Esta intervención será vital como se constata en correspondencia entre Bakema y Smithson: "Pete. Corbu is okey, we did win the battle against professors!"31. La alegría por la intervención de Le Corbusier muestra la importancia que aún tenía su opinión dentro de los CIAM. En el documento Le Corbusier, que por fin ha entendido que CIAM se encuentra en un momento de cambio, afirma que "les éléments établis par le Team X sont raisonnables et parfaitement acceptables" y que "j'ai relevé ceci, c'est que (question de jeunesse et d'inexpérience) des termes sont souvent mal employés", refiriéndose al documento Draft 5 elaborado por Smithson y que incluía muchas reivindicaciones de Orientation ${ }^{32}$. Giedion, que aún no confía en los jóvenes, escribirá: "Le Corbusier could not care a jot about CIAM $^{\text {,33. }}$.

La invitación al congreso la redactan Candilis y Le Corbusier ${ }^{34}$ según lo acordado. Se mantiene el método de trabajo propuesto por los Smithson (Scale of Association) y la idea principal del congreso es: "the function living becomes 'the habitat' when it is organically integrated in an environment"; ambos en la linea del Statement on Habitat de Doorn.

En la reunión previa al congreso, en septiembre de $1955^{35}$ se modifican las instrucciones redactadas por el Team10 en un "échange de vue entre les délégues des groupes et le Team X", eliminando la idea de Fields of Association (reacción molesta de Smithson) ${ }^{36}$. En segundo lugar, se decide el tema del CIAMX, que será "L'Habitat, Probleme de Relations. Premiere Propositions", como había sugerido Le Corbusier, y se cambia así el enfoque del diagrama de Geddes a la importancia de "las relaciones" como factor irrenunciable para una arquitectura más humana. También se pospone un año el congreso para desarrollar las instrucciones, y se modifica el lugar de celebración a Dubrovnik. "Les délégués présents sont unanimes pour remercier le Team X de sa contribution et du travail qu'il a fourni jusqu'ici".

La tarea para Dubrovnik era "to give form to the relations between functions, and to the more complex human structure of the agglomeration", y aunque el Team10 creía que esas relaciones solo se podrían definir tras el congreso $^{37}$, Giedion realiza una intervención en la que empatiza con las reivindicaciones de los jóvenes y da

\footnotetext{
28 (FLC D3-7 128)

29 (Candilis to Bakema, 9 May 1955)

${ }^{30}$ (FLC D3-7 30-33)

31 "Candilis told me that Corbusier was in complete agreement with our proposals for CIAM X" (ET10 44)

${ }^{32}$ El documento aprobado por Le Corbusier abandona la crítica a los CIAM y en su lugar incluye el relato histórico redactado en Orientation. Aunque no elimina la Scale of Association, sí que introduce las ideas de Change, Relationship between things, Doorstep y el Ecological Approach propuestas por el Dutch Group.

${ }^{33}$ (CIAM 42-JT-13-463)

${ }^{34}$ (FLC D3-7 95-97)

${ }^{35}$ (FLC D3-7 50-54)

${ }^{36}$ Circular a Sert y Team 10. "By the elimination of the 'Scale of Association', the emphasis on the community as an entity has disappeared, and with it the possibility of finding the key to Habitat which is the basic unifying belief of Team X'. (ET10 54-56)

37 (FLC D3-7 57-63)
} 
algunos ejemplos ${ }^{38}$. Entendiendo sus ideas, hace explícito su apoyo. Giedion habla de la necesidad de establecer relaciones entre funciones (algo que nunca había admitido) que serán más importantes que las funciones en sí mismas (calcado a las afirmaciones del Dutch Group). Se observa la inspiración de Giedion en el documento Orientation, y es evidente la simpatía de Giedion por Van Eyck, incluso en la manera de redactar y las expresiones utilizadas. Ha terminado por imponerse el enfoque de Orientation, y Smithson queda aún más alejado del núcleo de los CIAM.

Las preparaciones del congreso continuarán gracias a la intervención de Le Corbusier, no exentas de distintos puntos de vista, con los jóvenes intentando que sus ideas no desaparezcan de las instrucciones a los grupos y el consejo imponiendo elementos programáticos. Finalmente el congreso se organizará en cuatro sub-comisiones: clúster (tiene que ver con la idea de doorstep), mobility, change and growth, urbanism and habitat (the ecological aproach); similares a las propuestas por los holandeses.

\section{El mensaje de Le Corbusier: un problema de dos generaciones}

El 23 de Julio de 1956, poco antes de la celebración del décimo congreso, Le Corbusier redacta el "Message de Le Corbusier adressé au X Congrès CIAM à Duborvnik"39 y lo envía a Sert para que lo comparta con todos los presentes acompañada de una carta personal ${ }^{40}$ que resulta muy esclarecedora.

En la carta privada Le Corbusier sugiere que tras el congreso se disuelvan las comisiones creadas en Bérgamo y se modifiquen los estatutos que organizan los CIAM en grupos nacionales. Le Corbusier cree que el término nacional provoca "rivalité ou d'idées préconçues", y "conflits de préséance ou de jalousie"; y piensa que eliminándolo se abriría la organización a "la participation de nouvelles forces ou de nouvelles conceptions", grupos locales más diversos. La decisión de Bridgewater sirvió para distinguir "entre les travailleurs efectifs, et les verbeux, les éternels mécontents, les protestataires...", y ahora la metamorfosis debe operar sobre la base de la nueva generación. Para él los CIAM no son una cuestión de orden geográfico sino más bien de caracteres, de individuos "éminemment désintéressé" que asumen "les taches et n'ont jamais reserché des avantages personnels". Su opinión marcará la nueva dirección que toman los CIAM a partir de 1956, volviendo a ser una organización compuesta por individuos.

El mensaje público a los presentes en Dubrovnik es un precioso documento en el que Le Corbusier expresa su total apoyo a la nueva generación, tratando de definir su tarea. Se organiza en tres partes: “crise ou evolution? réponse: problème de générations", "la génération de 1928" y "la génération de 1956 va prendre le commandement".

\footnotetext{
${ }^{38}$ Giedion expone muchas de las ideas del Team 10, incluso de manera más clara: "the town is essentially relations, encounters, the coming together of you and me", "the age of compartmented specialization nears its end", "to study the new forms of human relations is the business of the architect, and it is the task of CIAM" (FLC D3-7 55-56)

${ }^{39}$ (FLC D3-7 121-127)

40 "le X Congrès doit dissoudre les commissions", "la métamorphose CIAM 1956 doit s'opérer sur la base de la nouvelle génération", "on n'atteindra à des buts pratiques que par ce désintéressement total, absolu, irréductible" (FLC D3-7 118-120)
} 


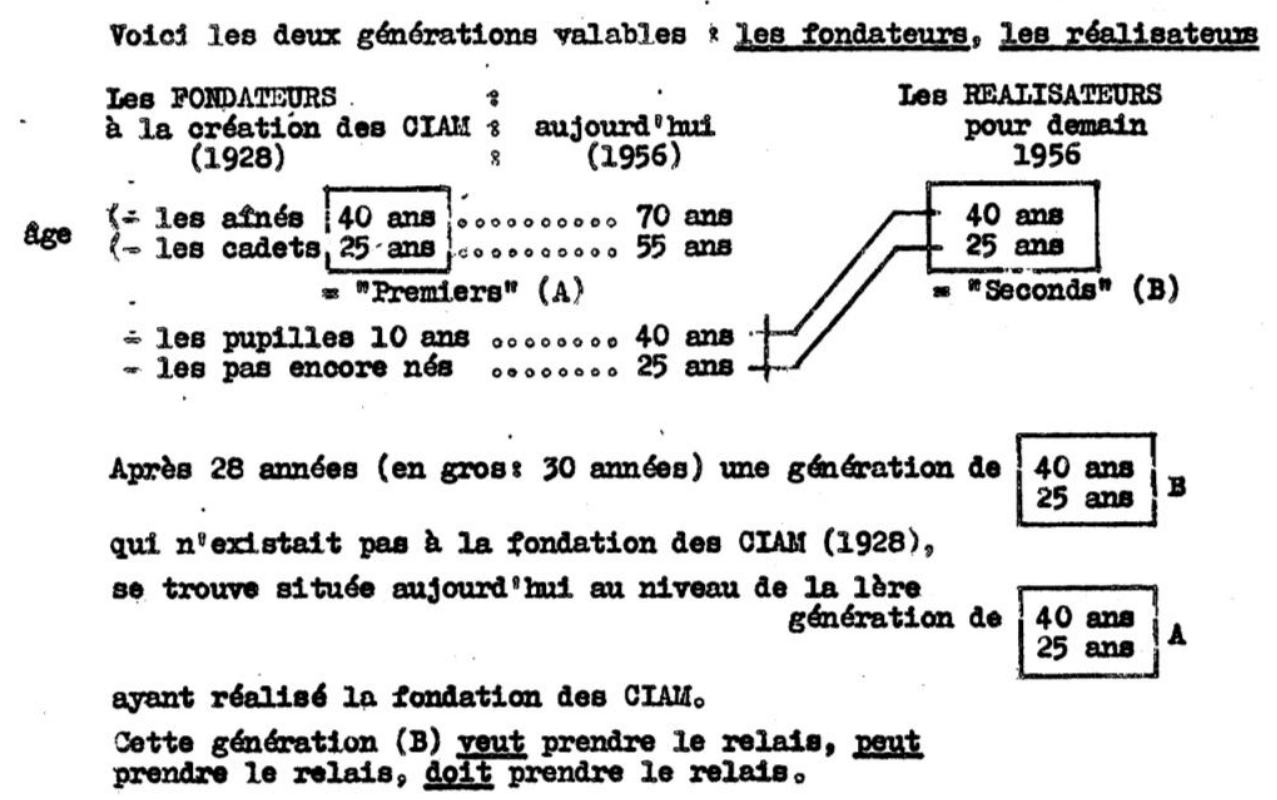

4. Fragmento del mensaje de Le Corbusier. CFLC-ADAGP

En la primera parte (Fig4) Le Corbusier resume el problema actual como un problema de generaciones: la de los fundadores, que son aquellos que en la fundación tenían 40-25 años (CIAM Premier); y la de los "realisateurs" que tienen ahora esa misma edad (CIAM Seconds). Es el momento de la nueva generación, que "veut prendre le relais, peur prende le relais, doit prende le relais". Le Corbusier no quiere eclipsarlos con su figura, "c'est pour cela que je ne suis pas à Dubrovnik", ya que la primera generación está "hors de l'ambiance de l'époque présente" mientras que la segunda generación se encuentra "ainsi en plein coeur de l'èpoque présente" y entiende los problemas actuales de la arquitectura. "Leus prédécesseurs n'y sont plus, ils en sont sortis, ils se trouvent hors des coups directs de la conjoncture".

En la segunda parte reitera el logro histórico de "les CIAM-PREMIERS", que han aportado "un programme cohérent du domaine bati, une doctrine" para que los CIAM Seconds la utilicen: las cuatro funciones (habitation, travail, loisirs y transport) y un método que tiene que ver con la estandarización y la normalización. Han instituido los CIAM, han enunciado los problemas de la arquitectura y el urbanismo, y han preparado la Carta de Atenas obteniendo reconocimiento mundial. La tarea de la segunda generación es "d'harmonisation", y esa armonización (habitat) se apoya sobre las ideas de los fundadores. 
1956, "Les CIAffoSECONDS" premnent le relais。

Deux graphiques valables peuvent résumer mon exposé :

ler graphique :

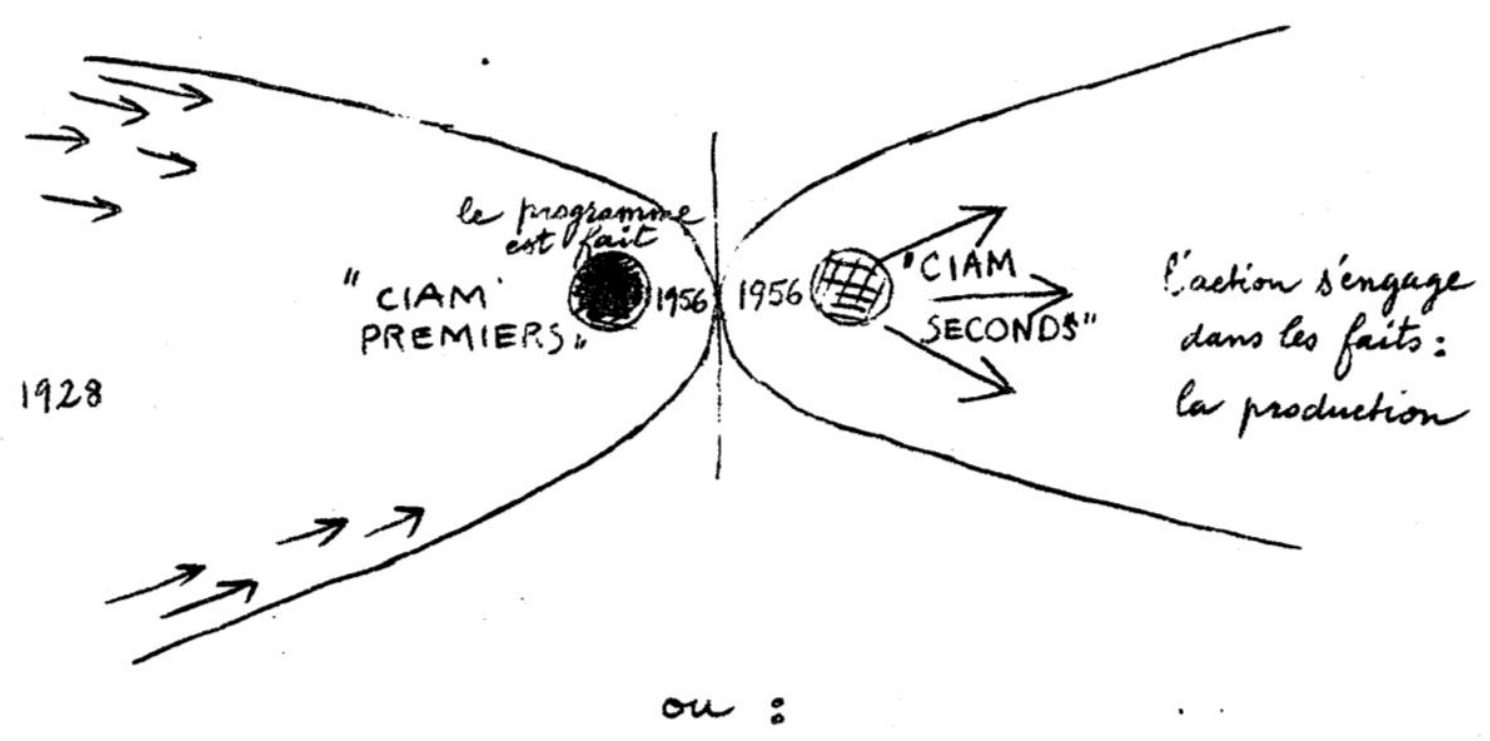

5. Fragmento del mensaje de Le Corbusier. CFLC-ADAGP

La tercera parte se resume en dos gráficos (Fig 5 y 6 ). En ellos Le Corbusier representa por primera vez de forma explícita el momento de inflexión en el que se encuentran los CIAM, demostrando que entiende la importancia de Dubrovnik. En el primer dibujo retoma la idea ya expresada: la primera generación construyó el programa y ahora la segunda generación debe demostrar con sus acciones (su tarea es la producción). En el segundo especifica: una doctrina de los CIAM Premiers que viene de lo social, la economía y la técnica (pone orden a la confusión), y una acción de los CIAM Seconds que buscará la armonización en esos mismos términos. 

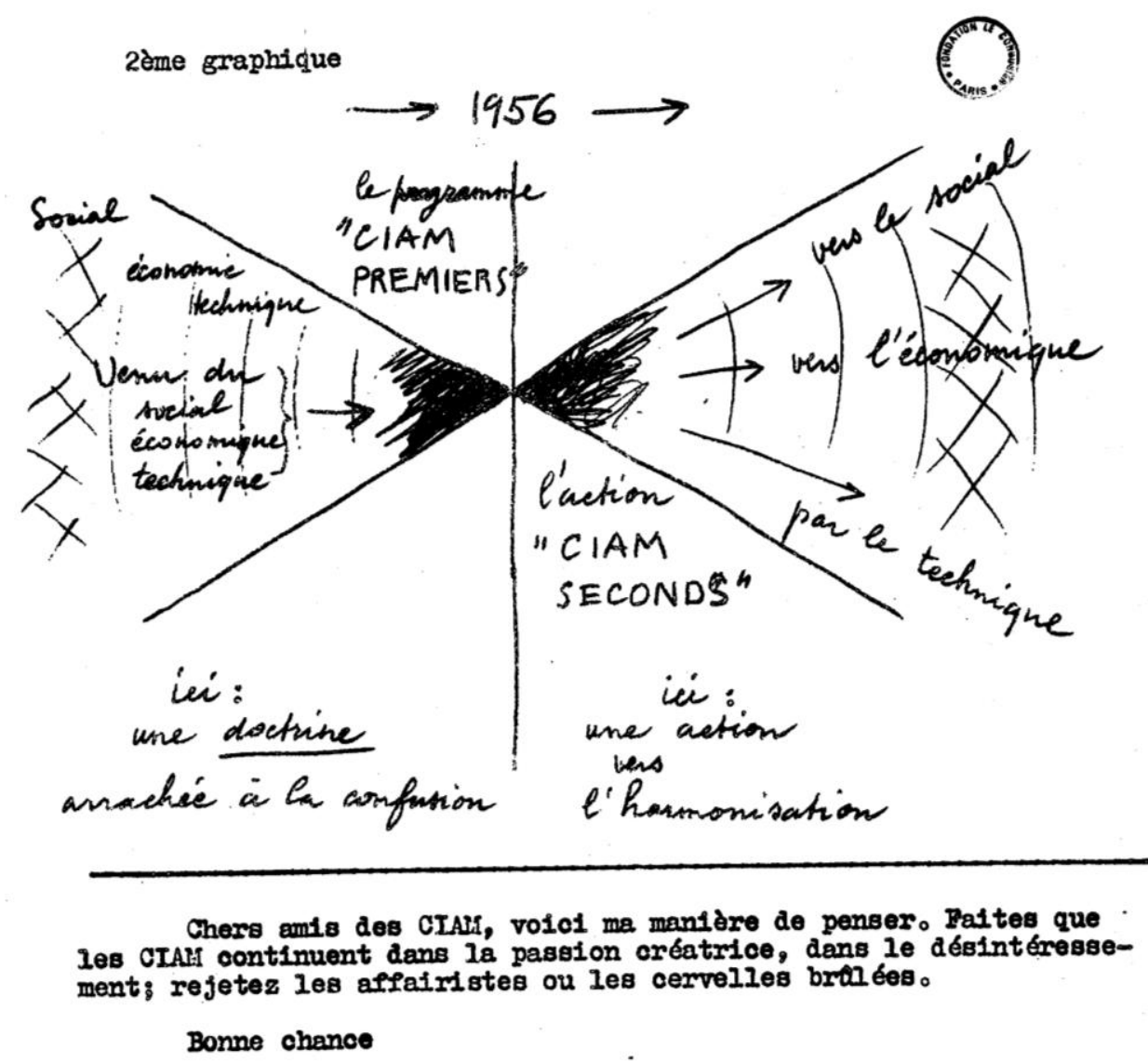

Vivent les CIAR-SECONDS !

6. Fragmento del mensaje de Le Corbusier @FLC-ADAGP

El mensaje termina. "Chers amis des CIAM, voici ma manière de penser. Faites que les CIAM continuent dans la passion créatrice, dans le désintéressement; rejetez les affairistes ou les cervelles brulées. Bonne chance. Vivent les CIAM-SECONDS!". Le Corbusier no pretende comprender todas las propuestas de los jóvenes pero entiende lo fundamental: la importancia de armonizar, relacionar las funciones entre sí para construir una arquitectura y un urbanismo más solidarios, pero sobre todo que es el momento de dejar que construyan su camino.

En el anexo, Le Corbusier insiste en la necesidad de elaborar una carta del Habitat que debe ser tarea exclusiva de los CIAM Premiers y pide a los presentes que eviten la discusión en torno a las generaciones: "Chers amis, cessez cette controverse, je vous en supplie". Le Corbusier es el primero de los fundadores en apoyar a los jeunes, y su mensaje, junto a la renuncia de su papel principal en los congresos, desemboca en la asamblea en Dubrovnik en la que se decide el proceso de reorganización de los CIAM. A partir de este momento el papel de Le Corbusier será oficialmente secundario, aunque seguirá manteniendo correspondencia con miembros del Team10. No volverá a tomar parte en las reuniones. 


\section{La reorganización de los CIAM: "batalla" en Otterlo}

La asamblea general tras el congreso de Dubrovnik, el 10 de agosto de $1956^{41}$, acuerda la reorganización total de los CIAM en los términos planteados por Le Corbusier y encarga la tarea a Bakema, Emery, Howell, Rogers, Roth, Smithson y Woods. En enero se disolverían los grupos y los CIAM volverían a estar compuestos por individuos. Se envía un cuestionario a todos los delegados para que propongan a 30 individuos que formarán los CIAM. Muy pocos delegados responden al cuestionario, entre ellos Le Corbusier ${ }^{42}$. Giedion sugiere ${ }^{43}$ que se utilice otro nombre para la nueva etapa de los CIAM, pues es la única manera de conseguir una verdadera renovación.

También responde Bakema ${ }^{44}$ en nombre del Dutch Group, Candilis y Woods, que aprovecha para hacer una serie de propuestas. Cree que una organización como CIAM carece de sentido si los miembros no admiten que hay problemas imposibles de solucionar individualmente, y que los principios de la arquitectura moderna no se pueden desarrollar sin reconocer su relación con el "total human habitat" (diagrama de Geddes). Hace falta reconocer en el mismo proceso la investigación, el diseño, el uso, la realización y la eliminación. "Recognition of the totalness of this process can result in an architecture called total arquitecture as a further step to the expression of full life; life which is substituting the work 'to possess' by the word 'to use"'. Cree que los grupos deben tener especialistas de todo tipo y el contacto debe ser entre individuos. Las reuniones deben realizarse siempre en lugares en los que se puede visitar obras construidas (esta será una de las premisas de las reuniones del Team10). Sert, Gropius, Giedion y Tyrwhitt ${ }^{45}$, en su carta conjunta, proponen a Bakema como presidente del nuevo CIAM.

En el grupo inglés se han sucedido una serie de acontecimientos. Según P. Smithson ${ }^{46}$, el resultado más positivo del décimo congreso es que los miembros han empezado a dudar de la existencia de los CIAM. En Inglaterra supuso la disolución del Mars Group, y la posibilidad de construir un nuevo sistema de contactos desde cero sin arrastrar acuerdos pasados. En una carta a Bakema y Woods ${ }^{47}$, Smithson va un paso más allá afirmando que se opone completamente a la elección de 30 miembros sin que se decida en un congreso quién tiene el espíritu para formar parte de la nueva organización. En una carta el 9 de Diciembre ${ }^{48}$ al consejo de los CIAM, Alison y Peter Smithson se muestran claros en su postura: "We must make a complete break with the name CIAM". En su opinión el nombre representa una estética que está acabada y aunque creen en el objetivo de la organización, no creen en sus métodos (pensamiento diagramático, planeamientos cartesianos, propiedad pública del suelo...) "It would be better to make CIAM history and start a new group". Frente al "CIAM de los 30" opinan que a cada reunión debe asistir un grupo de personas distinto.

\footnotetext{
${ }^{41}$ Al finalizar CIAMX se reúne la asamblea como había sugerido Le Corbusier. En el documento hay una anotación a mano de Le Corbusier: "y mai pas sur le nom CIAM!". Puede que el nuevo CIAM no se llame CIAM, uno de los temas de mayor discusión durante el proceso de reorganización. 


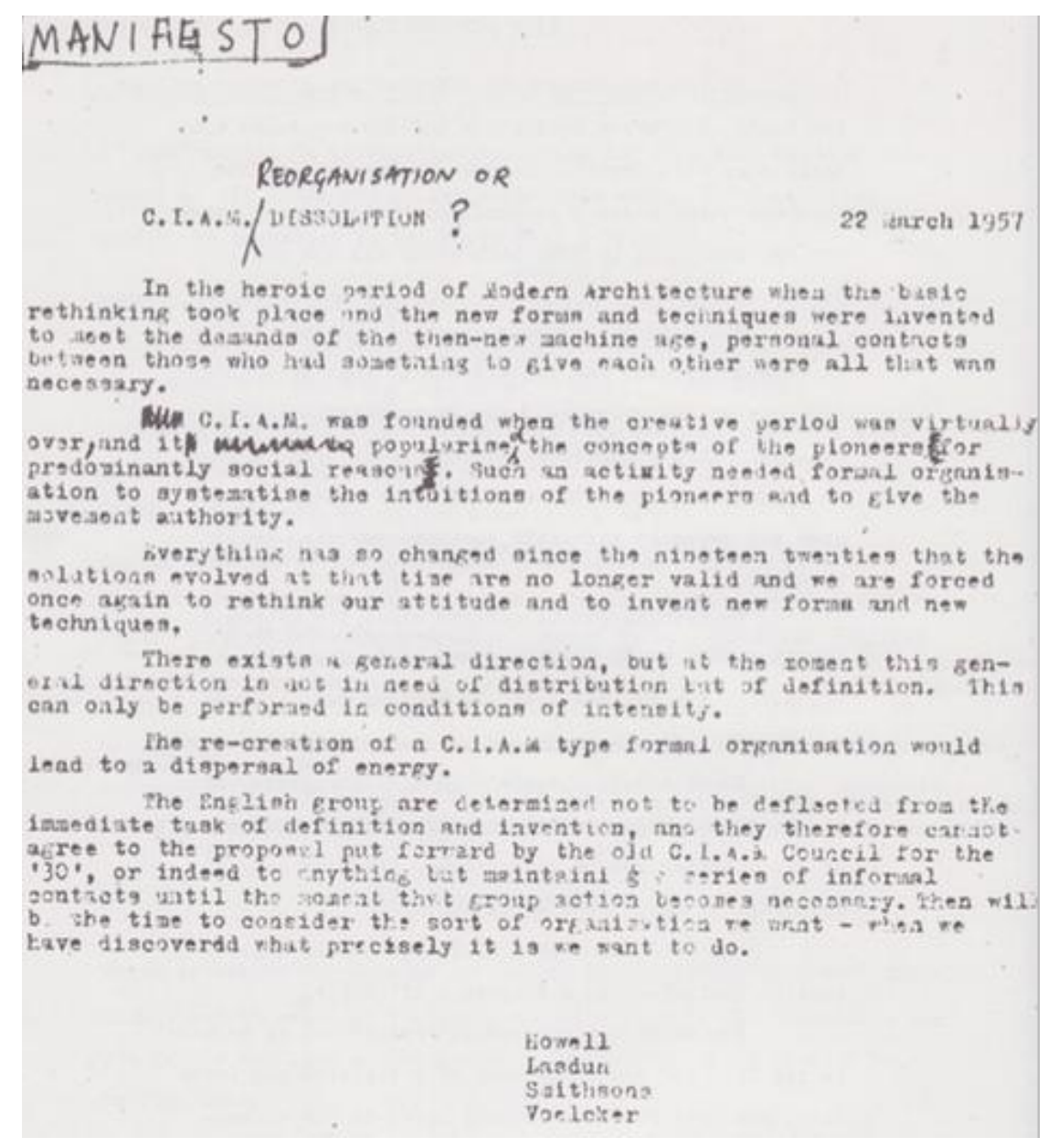

7. CIAM Dissolution (ET10 77) OFLC-ADAGP

Los comentarios son ignorados y los Smithson elaboran un documento titulado CIAM Dissolution (Fig7) en el que concretan su punto de vista: "the English group are determined not to be deflected from the immediate task of definition and invention, and they cannot agree to the proposals by the old CIAM Council, or indeed to anything but maintaining a series of informal contacts". El documento es firmado también por Howell, Lasdun y Voelcker ${ }^{49}$ pero fue elaborado por los Smithson como demuestran las distintas versiones.

En paralelo se suceden las propuestas de Bakema que queda en una posición intermedia ${ }^{50}$ y prefiere un enfoque constructivo, similar al de Candilis-Woods ${ }^{51}$ y Soltan $^{52}$. Sin embargo, la actitud destructiva de los ingleses

\footnotetext{
${ }^{49}$ (ET10 78) (FLC D-3-19 118)

${ }^{50}$ Propone la organización de una reunión para tratar la disolución. Resume los puntos de vista: continuar; disolver y crear una red para contactos informales; o reorganizar (lista 30 miembros). Bakema apuesta por continuar con un espíritu de investigación e imaginación.

(FLC D3-19 106-107)

${ }^{51}$ CIAM no pude seguir siendo patrimonio de Le Corbusier, Giedion, Gropius... La tarea no consiste en distribuir las responsabilidades sino en hablar sobre los nuevos problemas y buscar soluciones. No importa el nombre sino la unión bajo el espíritu adecuado. "Dans tout les cas, nous continuerons à travailler dans l'esprit des CIAM, avec ou sans organisations". (FLC D3-19 122-124)

${ }^{52}$ Afirma que el nombre de la organización no tiene importancia. Pero una organización en la linea de CIAM debe existir para garantizar el contacto de arquitectos de todos los lugares.

(FLC D3-19 117)
} 
provoca una respuesta contundente. En mayo de 1957, Sert, Gropius, Giedion y Tyrwhitt envían una carta a Le Corbusier $^{53}$ avisando de que circulan documentos que hablan de la disolución de los CIAM. Según su versión, en Dubrovnik se votó cómo sería el nuevo CIAM por amplia mayoría. "The persons who have made this statement may have forgotten this, though some were elected to the Reorganization' Committee". Saben que es tiempo para un cambio, pero creen que no se puede mantener una actitud destructiva cuando han tomado responsabilidades. "It is not a question of abandoning the old, but of supplementing and transcending it".

Cuando Bakema pide más tiempo para desarrollar las ideas del grupo y decidir la reorganización, el consejo responde enfadado: "the Reorganization Commitee has not carried out the committments",54, y sugieren una reunión para decidir el futuro de los CIAM que se celebrará en la Sarraz. Otros, como P. Emery, señalan directamente a Peter Smithson ${ }^{55}$ como el causante de la disputa y expresan su molestia por la intención destructiva de los ingleses que sin tener en cuenta la decisión mayoritaria en Dubrovnik dan publicidad a ideas desestimadas.

\section{La Sarraz 1957: “C'est bien!”}

En septiembre se celebra en La Sarraz una reunión para aclarar las distintas opiniones que se habían hecho públicas. Los Smithson, por carta ${ }^{56}$, renuncian a asistir a la reunión para expresar su enfado: "one can only recreate what one loves by repudiating it". Adjuntan una propuesta en la que ya no hablan de disolución (cambian de idea gracias a Bakema) pero siguen rechazando la elección de 30 miembros. Proponen un nuevo nombre: CICON (CIam CONtinuity).

En La Sarraz ${ }^{57}$ se disuelven definitivamente los grupos. Como apuntaba Le Corbusier (y los jóvenes desde Doorn), era necesario restringir los CIAM a aquellos miembros que fueran activos. Se nombra un nuevo comité en el que se sustituye a Smithson y Howell por Voelcker y se adopta un nuevo nombre, "CIAM: Research Group for Social and Visual Relationships", en sintonía con las ideas del Dutch Group. La tarea del comité es designar los nuevos participantes en base a las recomendaciones de los grupos y organizar el próximo congreso en el que se decidirá el rumbo que toman los CIAM.

Un mes después Bakema pide ${ }^{58}$ a todos los delegados que envíen la lista de nombres. En la carta menciona los objetivos del nuevo CIAM: la necesidad de desarrollar el arte de vivir (término que atribuye a Le Corbusier en carta de 1953) pues todas las actividades humanas se realizan en espacios; la importancia de introducir la arquitectura y el urbanismo como elementos activos dentro de la sociedad y la relación entre las funciones. La carta aparece anotada a mano por Le Corbusier (“C'est bien!") y él mismo contestará personalmente a

\footnotetext{
53 (FLC D3-19 96-98)

${ }^{54}$ Carta de Giedion, Sert, Gropius y Tyrwhitt (FLC D3-19 100)

${ }^{55}$ P. Emery a Bakema el 25/06/1957

"Les réponses sont parfaitement encourageantes, à l'exception d'une seule, celle de SMITHSON qui, bien que membre du Comité, et ayant accepté les décisions de Dubrovnik, revient sur toutes ces décisions, et demande la dissolution". "Je pense que es faisant, HOWELL, LASDUN, SMITHSON et VOELCKER perdent toute qualité pour discuter de l'avenir des CIAM car on ne peut charger quelqu'un de crééer ou de protéger ce qu'il a formellement l'intention de détruire".

(FLC D3-19 101-103)

${ }^{56}$ (ET10 80-81)

${ }^{57}$ (FLC D3-19 125-127)

58 (FLC D3-19 128-129)
} 
Bakema $^{59}$. A partir de este momento Bakema informaría directamente a Le Corbusier con copias de los documentos anotadas $^{60}$. Le Corbusier y el Team10 quedan así unidos (Fig8).

Le Corbusier a Bakema ${ }^{61}$ :

Merci de vos 'Directives pour la...

Tout cela me parait très bien. Il faut que la vie passe avant tout et les feuilles du printemps s'ouvrent devant les feuilles de l'automne de l'année précédente qui se replient tranquillement et parfois tombent,

Bon courage! et amicalement à vous

Anotación de Bakema en respuesta ${ }^{62}$ :

Merci pour votre encouragement poétique. J'espère que l'arbre que s'appelle Le Corbusier restera fort enfin que ses feuilles donneront encore longtemps beaucoup de protection.

Merci Jeanneret

Tras la reunión en La Sarraz el comité se vuelca en su tarea de organizar el congreso, que será celebrado en Otterlo en septiembre de 1959. Finalmente se amplía el número de invitados a 50 (Le Corbusier rechaza la invitación) y se propone que las discusiones filosóficas y abstractas se eviten. El congreso se basará en el análisis de proyectos construidos ${ }^{63}$. El objetivo de la reunión de Otterlo es descubrir si existen unas ideas comunes a los participantes y decidir la continuidad de los CIAM. El tema propuesto está en completa sintonía con el Team10: la manera de organizar las relaciones entre el hombre y su entorno físico, unas relaciones que no son estáticas, sino que están sujetas a la evolución perpetua y el crecimiento continuo de los individuos. Si existe afinidad en este punto, CIAM podrá continuar. En el último documento oficial previo a Otterlo encontramos un párrafo que ilustra la concepción de la arquitectura de los miembros del comité (Fig9)

\footnotetext{
59 “J'ai reçu votre document CIAM du 22 octobre 1957 et je l'ai annoté: C'est bien!. Amicalement à vous" (FLC D3-19 130)

${ }^{60}$ Son numerosas los mensajes a Le Corbusier en notas a mano (FLC D3-19 132, 138)

${ }^{61}$ (FLC D3-19 145)

62 (FLC D3-19 146)

63 (FLC D3-19 132-137)
} 


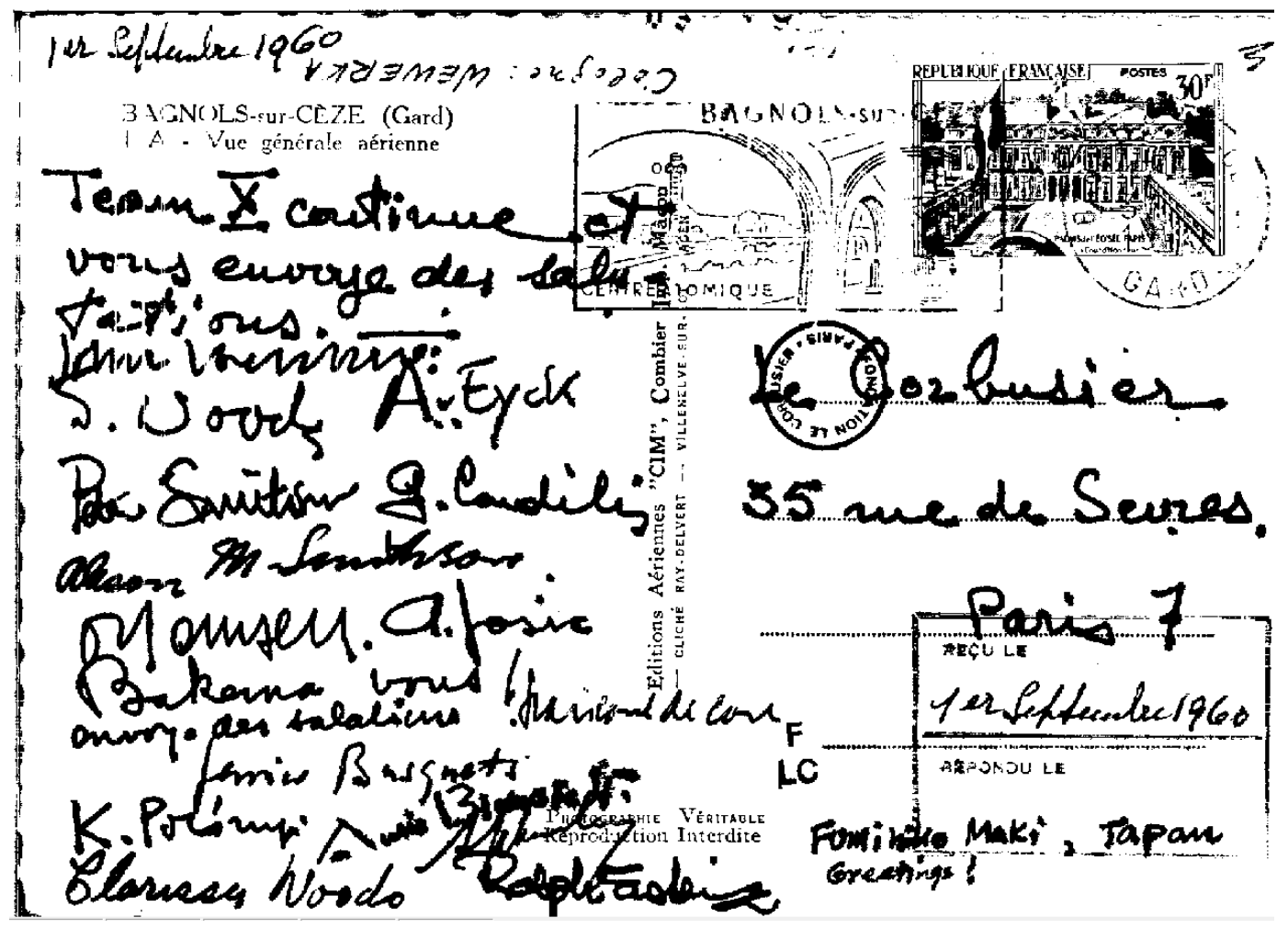

8. Postal de TeamX a Le Corbusier. (FLC D3-19 171-172) @FLC-ADAGP

2. That together with an explanation of their particular work, authors should give their general opinions on the present position of architecture and planning. Each author skould describe the factors which he considers to be fundamental Each author skould descrin which he is working. The co-ordinating group considers to the circumstances in which he is working. Thor could indicate how the spatial and plastic implications of his work might contribute to a more positive connection between people and their surroundings.

itive oonnitecture and planning are either passive, (the new towns, restrictive legislation etc.) or are used irresponeibly (Stalin Allee, advertising style etc.), at the present time. The force of architecture and planning is ignored by specialists in other disciplines. of architecture and planning isning are thought of as 'cake' and not as daily Unhappily

If architects are no longer able to act through their building, their identity will be lost, they will become: industrial designers, decorators etc. Architecture should be life-enhancing.

3. That, if some affinity of approach is apparent at Otterlo, the co-ordinating group suggests that C.I.A.M. should become a simple centre for the exchange of ideas and research in architecture and planning.

\begin{tabular}{|c|c|c|c|}
\hline . & & $\begin{array}{l}\text { Co-ordinating } \\
\text { Bakema } \\
\text { Rogers } \\
\text { Roth } \\
\text { Yoelcker } \\
\text { Wogenscky }\end{array}$ & $\begin{array}{l}\text { group: } \\
\text { - Netherlands } \\
\text { - Italy } \\
\text { - Switzerland } \\
\text { - England } \\
\text { - France }\end{array}$ \\
\hline oretariat: & $\begin{array}{l}\text { J.B. Bakema, } \\
\text { Posthobrnstraat } 12^{\text {B }} \\
\text { Rotterdam (Netherlands). }\end{array}$ & $\begin{array}{l}\text { Co-opted momb } \\
\text { Grung } \\
\text { Soltän } \\
\text { Traut rein }\end{array}$ & $\begin{array}{l}\text { bers: } \\
\text { - Norway } \\
\text { - Poland } \\
\text { - Germany }\end{array}$ \\
\hline
\end{tabular}




\section{La disolución de los CIAM: "pris la decision dans l'esprit de La Sarraz"}

Tras meses de preparación se celebra el primer congreso con la participación exclusiva de los jóvenes. Días antes Le Corbusier envía un telegrama (Fig10) a Bakema y le insta a tomar la decisión sobre el futuro de CIAM en el espíritu de la Sarraz (1928).

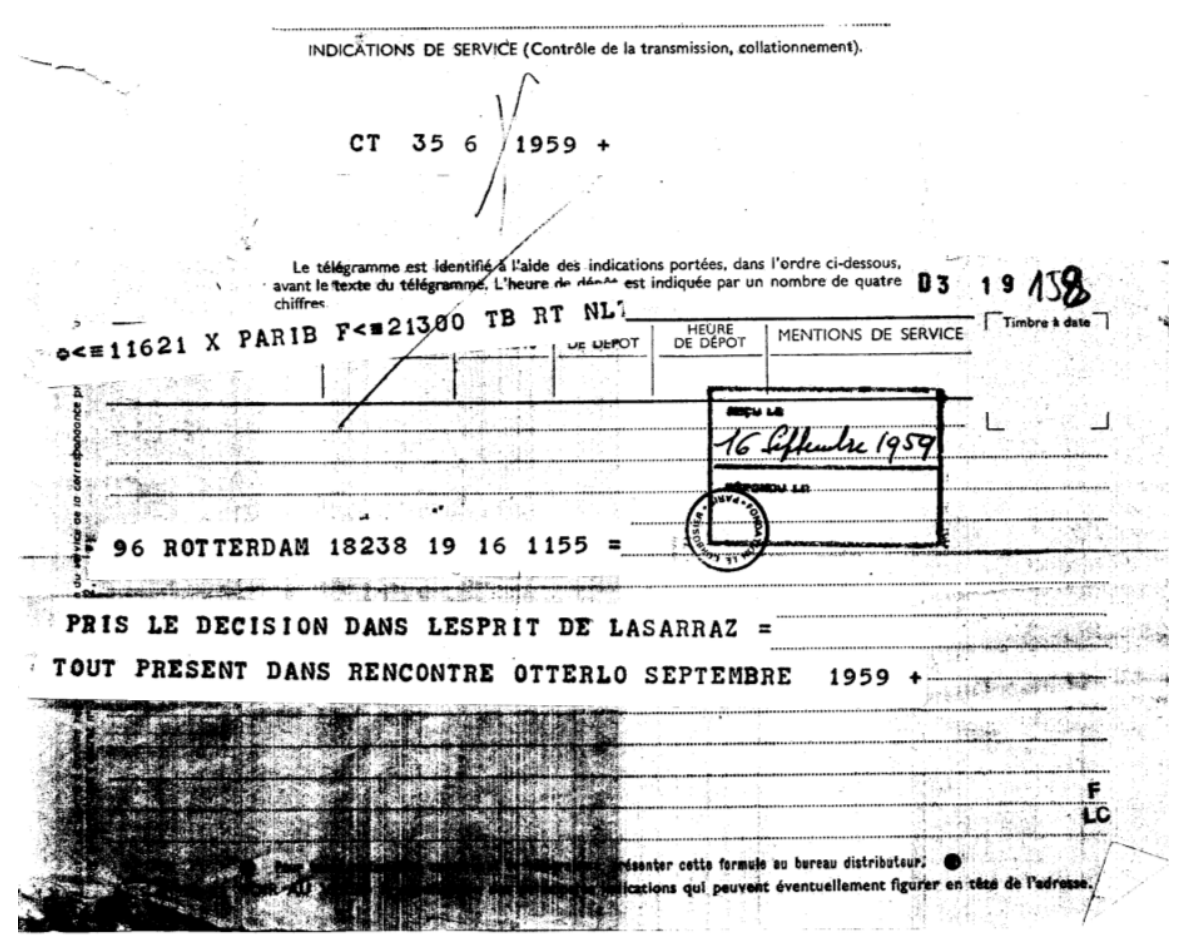

10. Telegrama de Le Corbusier a Bakema. (FLC D3-19 158) @FLC-ADAGP

Aunque podría pensarse que la disolución de los CIAM (Fig11) que se firmaría el último día del congreso fue un acto de "traición" de los jóvenes, la decisión se toma dans l'esprit de la Sarraz: construir a través de la unión de individuos un nuevo pensamiento para solucionar los problemas de la arquitectura. En el resumen de la reunión ${ }^{64}$ se constata la existencia de dos maneras de trabajar dentro de CIAM: una que se puede describir neutral y en la linea de las propuestas de CIAM desde 1928, y otra que puede considerarse agresiva, que intenta trabajar con cualidades individuales y sociales para desarrollar un nuevo lenguaje coherente con el comportamiento humano (la mayoría elaborados por miembros del Team10). Debido a la falta de coherencia en los planteamientos, se decide que "the name of CIAM will be used no more in relation to future activities of the participants", pero se constata el deseo de comparar el resultado de las actividades futuras y se crea el Post Box for the development of the Habitat del que se encarga Bakema (Fig12).

${ }^{64}$ (FLC D3-19 159-160) 


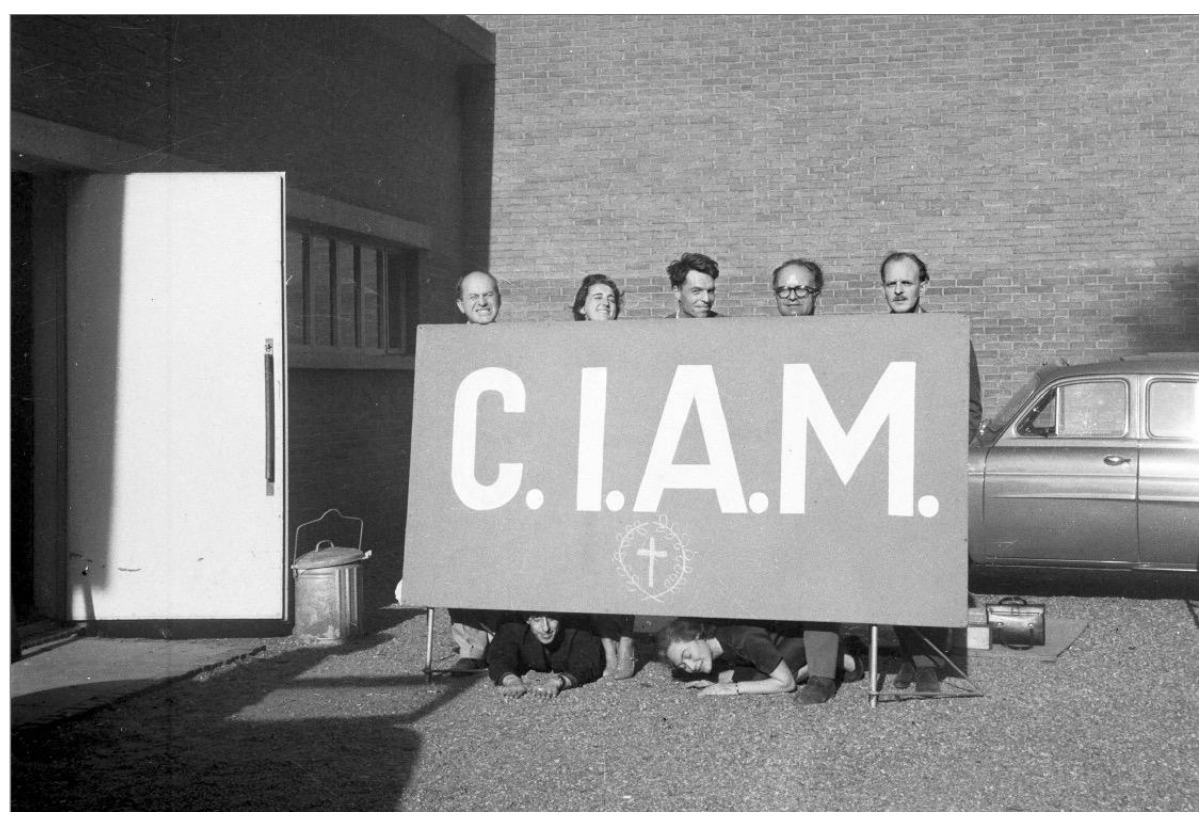

11. Fotografía de CIAM Otterlo. Alison und Peter Smithson Archiv Nederlands Architectuurinstituut

Esta decisión en ningún momento implica un rechazo de la tarea previa de los CIAM que en el mismo documento alaban. De hecho, la decisión implica simplemente asumir que la organización llamada CIAM ya no es útil para desarrollar la idea de habitat, pero los individuos del Team10 seguirán trabajando con el mismo espíritu. Habría que preguntarse si la decisión de romper con los CIAM no responde al afán de protagonismo de algunos participantes.

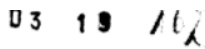

$-2-$

Now, I make the next propositions:

1. If you are confrontated with problems concerning Habitat and you like to confrontate them with other colleaques, who are working in research spirit, you send me plans, text and photos concerning this problem.

2. I shall prepare a folded illustrated communication paper with summeries of this information.

3. After a period there will be a meeting of those whonge information, organized by themselves.

4. The name of thiscrchange centre will be :

Post Box for the development of Habitat.(B.P.H.)

Borte Postale pour le developpement de l'Habitat.(B.P.H.)

Briefkasten für die Entwicklung von Habitat.(B.P.H.)

5. The aim will be to develop architecture and townplanning towards a language which can communicate about humain behaviour.

$$
\begin{aligned}
& \text { B.P.H. } \text { 20th. of September } \\
& \text { Rotterdam } \\
& \text { Posthoornstraat } 12^{\mathrm{B}} \\
& \text { J.B. Bakema }
\end{aligned}
$$

12. Fragmento del comunicado que anunciaba la disolución (FLC D3-19 162) CFLC-ADAGP 


\section{Team 10: "To be alone, to be together"}

La ruptura de los CIAM no está exenta de polémica. En agosto de $1960^{65}$, tras la reunión en Otterlo, Giedion envía una carta a Le Corbusier firmada por Sert, Gropius, y Giedion. Comienza narrando la fundación de los CIAM y enumerando sus logros, pues los jóvenes estaban siendo muy injustos y no estaban agradeciendo el trabajo de los fundadores. Según su versión los fundadores decidieron apartarse en Aix para pasar el relevo a los jóvenes. Allí se presentaron varias posibilidades: abrir una nueva página, como proponía Le Corbusier (CIAM Seconds), o eliminar los CIAM como proponía Giedion. Se decidió por unanimidad continuar por insistencia del Team10. En Otterlo un grupo minoritario entregó a la prensa un comunicado en nombre de los CIAM (Fig10) en el que se decidía disolverlos sin haberlo discutido en común, por lo que no podía considerarse un consenso de la organización. "Repeated attacks on the leadership and policies of the previos CIAM" han causado esta ruptura. Aún así CIAM ya ha cumplido su tarea y depende de los jóvenes presentar una plataforma constructiva que siga atrayendo a arquitectos de todo el mundo.

Ante el silencio de Le Corbusier, Giedion vuelve a escribirle ${ }^{66}$ para publicar la carta con su nombre. Le Corbusier contestará finalmente con ironía y enfado, pues cree que los jóvenes han tomado la decisión correcta (Fig13). Aún así, la carta de Giedion/Sert/Gropius se publicará bajo la firma de Le Corbusier.

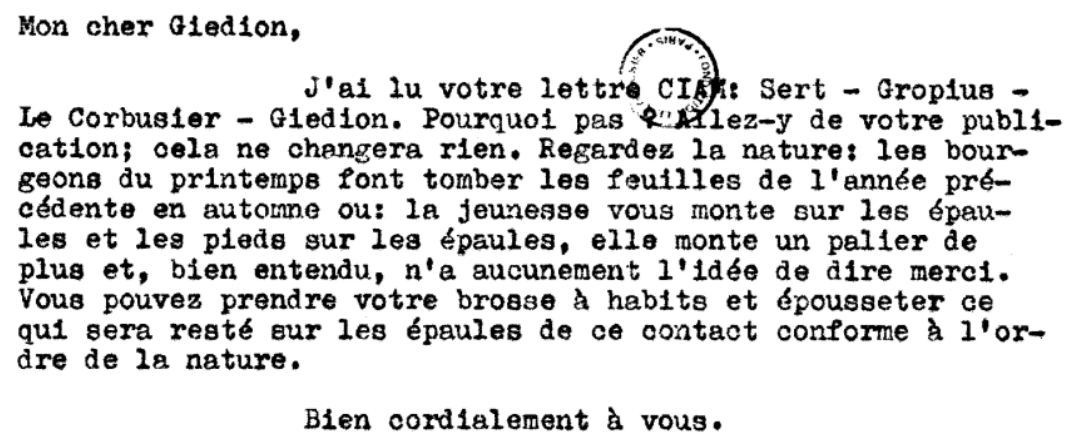

LE CORBUSIER

\section{Respuesta de Le Corbusier a la carta de Giedion. (FLC D3-19 177) @FLC-ADAGP}

Dolido por la misiva, Bakema contesta. El comunicado de Otterlo fue aceptado por todos los participantes en una discusión abierta la última jornada del congreso. En su opinión, aunque fuera cierto que Team10 anunció la disolución, no habría sido posible mientras quedasen miembros que quisieran continuar. Se decidió dejar de usar el nombre CIAM porque los objetivos de 1960 no son los mismos que los de 1920, y el nuevo nombre se elegirá

\footnotetext{
65 "there was an organized opposition against any positive statements about reorganization and continuity by a small group (Team10) whose basis attitude was negative" 
cuando los nuevos objetivos hayan sido definidos. En la copia que Bakema envía a Le Corbusier ${ }^{67}$, escribe una anotación a mano:

La vérité d' Otterlo n'est pas dans la lettre circulaire de Giedion.

Je crois qu'avec cette lettre l'histoire CIAM est compromis. Jamais une organisation ne peut faire de l'architecture. C'est l'architecture qui organise.

Bak

Para Bakema el Team10 no sustituye a los CIAM, sino que es uno de los grupos que decide trabajar de manera independiente, "perhaps others will follow". Si los CIAM deben o no continuar es una pregunta que deben hacerse sus miembros, y la decisión del Team10 no tiene nada que ver con su disolución, sino con la informalidad fundamental para su concepción como grupo creativo. Van Eyck y Bakema son tajantes ${ }^{68}$ :

The social responsibility which characterized the activities of CIAM's first years, formed its specific morality, but faded as the years went on. What had characterized CIAM in the beginning was all but lost. This fact was reflected in CIAM's static institutional form, in its arbitrary commissions and method of study...

We believe in architecture which can express human feelings of all kind.

To be alone, to be together, with technic, without tecnhic, under the trees, over the trees, on a hill or in a valley, to be child, to be old, above and below, soft and hard.

There is no hierarchy.

Our urban districts could surprise and stimulate again if the hidden potency of our new social structure (open society) should be expressed by building.

The architect would be indispensable again and architecture could be a function realizing everybody's right to full life.

Quizás no fuera tal la ruptura. Puede que en realidad siempre buscasen recuperar el espíritu de los primeros CIAM, y que la única forma fuera romper con una organización que se había vuelto dogmática y se había visto excesivamente jerarquizada. Con estas palabras comienza el camino del Team10 en solitario, en total sintonía con los primeros CIAM.

\footnotetext{
${ }^{67}$ Circular de Postbox (FLC D3-19 178-181)

${ }^{68}$ Extracto de circular: "Les informations du rencontre Otterloo données para un document, si que par vous. Gropius, Giedion, Sert ne sont pas juste"

(FLC D3-19 182-183)
} 


\section{El último mensaje de Le Corbusier}

El 5 de enero de 1961 los jóvenes publican el TeamX Statement, en el que rompen por completo con los CIAM y expresan su gratitud a los que han mantenido tantos años la plataforma. "Nous continuerons nos recherches et espérons de confronter les résultats avec les travaux des autres" ${ }^{69}$, dice Bakema a Le Corbusier.

Le Corbusier, generoso, enviará las últimas palabras de apoyo al grupo (Fig14), que serán muy bien recibidas y agradecidas por Bakema (Fig15).
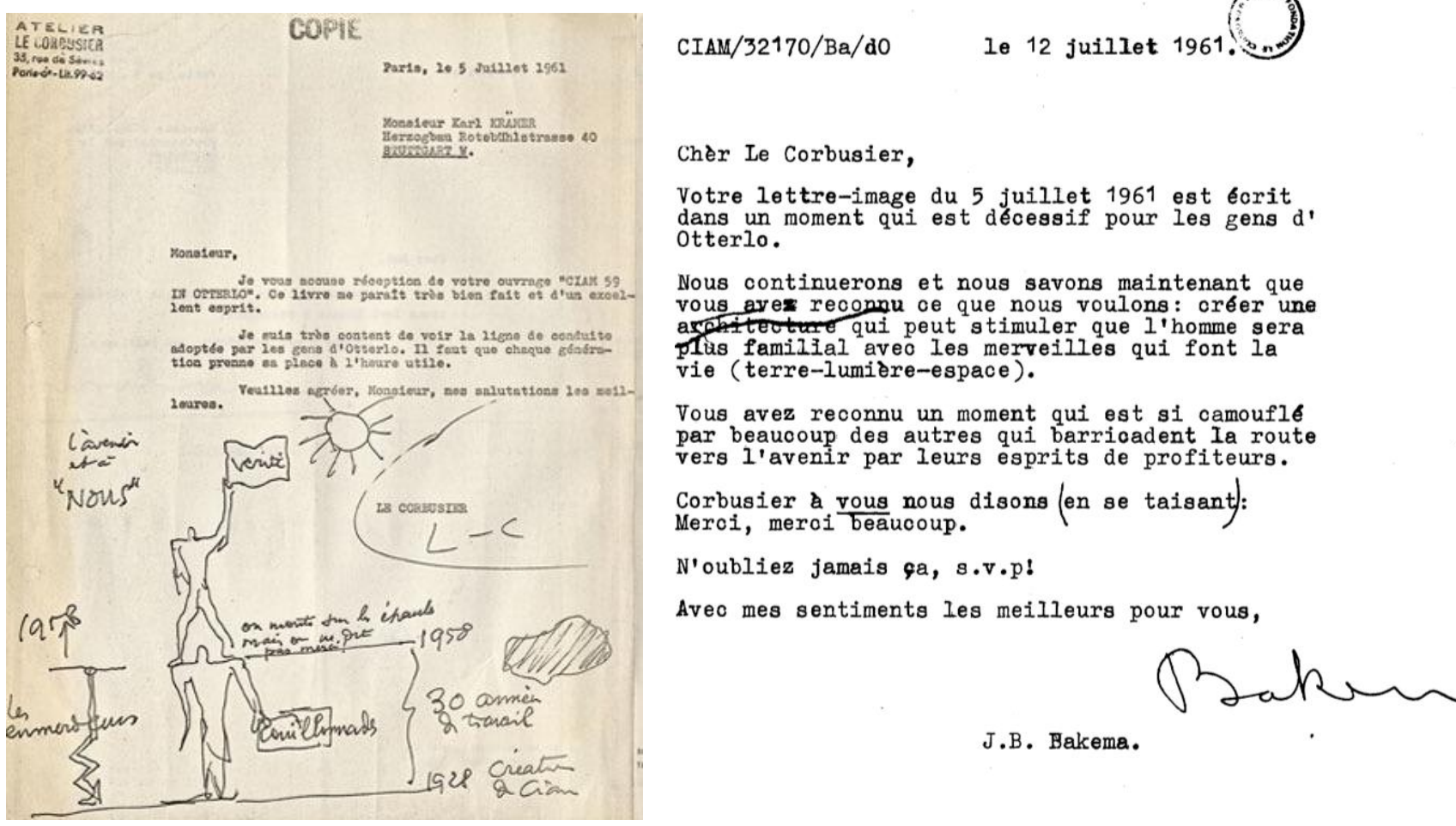

J.B. Bakema.

14. Dibujo de Le Corbusier enviado a Bakema. (FLC D3-19 186) OFLC-ADAGP

15. Agradecimiento de Bakema a Le Corbusier. (FLC D3-19 190) @FLC-ADAGP

Todos estos contactos a lo largo del tiempo muestran el importante papel de Le Corbusier en el nacimiento del Team 10, bien sea como espectador pasivo o como incitador de unas ideas menos dogmáticas; aunque finalmente fuera la inmovilidad de la segunda generación la que produjo la ruptura. Sin embargo, Aldo van Eyck recordaba también con admiración los encuentros en el apartamento de Le Corbusier, en los que el maestro participaba activamente conversando y dibujando con tiza y borrador, haciendo de moderador en las discusiones, y señalando, cuando la conversación se estancaba, otros puntos de vista que permitían continuar y enriquecían las conclusiones obtenidas. Bakema nos deja notas y cartas de agradecimiento sincero, y Candilis numerosas muestras de aprecio. Enormemente generoso en su actitud y tan inteligente para apartarse a tiempo y dejar paso a los que tenían la fuerza e ilusión de la juventud, que en su momento él también había tenido. Un preciso acto de identificación y legado.

\footnotetext{
${ }^{69}$ (FLC D3-19 181)
} 
En una de las más importantes reuniones del Team 10, en Royaumont 1962, los CIAM Seconds, que habían tomado el relevo, sintetizan en un mensaje a Le Corbusier el agradecimiento de toda una generación: "Nous Continuons, Merci". Ese agradecimiento que no puede expresarse de otra manera, sino únicamente diciendo: gracias, no te defraudaremos (Fig16).

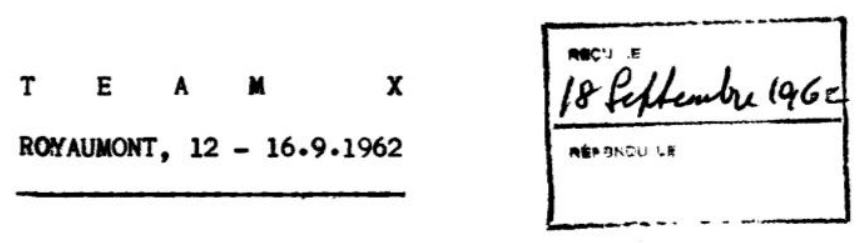

A L'ATTENTION DE M. LE CORBUSIER.-

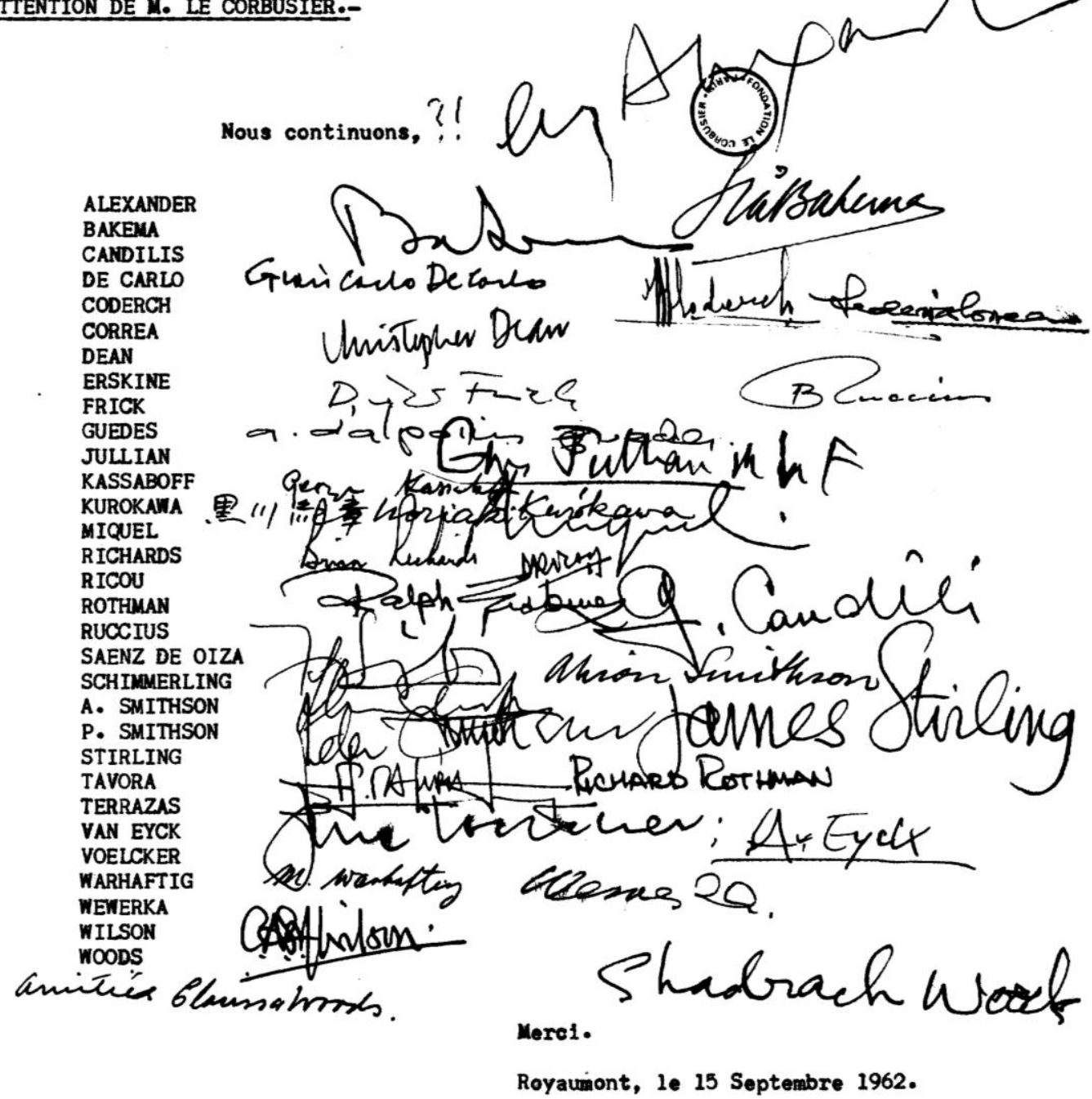

16. Carta de agradecimiento a Le Corbusier. (FLC D3-19 210) OFLC-ADAGP 


\section{Bibliografía}

Archivo: Fondation Le Corbusier, Paris.

Newman, Oscar, CIAM '59 in Otterlo. New York: Universe Books Inc, 1961

Pedret, Annie. CIAM and the emergence of Team 10 thinking 1945-1959. Director: Mark Jarzombek.

Massachusetts Institute of Tecnhology, Department of Architecture, 2001

Risselada, M. and Van den Heuvel, D. Team 10: in search of a Utopia of the present. Netherlands: NAI Publishers, 2006

Roth, Alfred. The New Architecture. Zurich: Les Editions A'Akrchitecture, 1940

Smithson, Alison. Team Ten Meetings, 1953-1981. United States: Rizzoli International Publications, 1991

Smithson, Alison. The Emergence of Team 10 out of CIAM. London: Architectural Association, 1982

Van Eyck, Aldo. Collected Articles and Other Writings 1947-1998. Amsterdam: Vincent Ligtelijn y Francis Strauven, SUN Publishers, 2008 